\title{
Combined Reporter Gene PET and Iron Oxide MRI for Monitoring Survival and Localization of Transplanted Cells in the Rat Heart
}

Takahiro Higuchi*1, Martina Anton*2, Katja Dumler ${ }^{2}$, Stefan Seidl ${ }^{3}$, Jaroslav Pelisek ${ }^{4}$, Antti Saraste ${ }^{1}$, Andrea Welling ${ }^{5}$, Franz Hofmann ${ }^{5}$, Robert A.J. Oostendorp ${ }^{6}$, Bernd Gansbacher ${ }^{2}$, Stephan G. Nekolla ${ }^{1}$, Frank M. Bengel ${ }^{1}$, Rene M. Botnar ${ }^{1}$, and Markus Schwaiger ${ }^{1}$

${ }^{I}$ Nuklearmedizinische Klinik und Poliklinik, Technische Universität München, Munich, Germany; ${ }^{2}$ Institut für Experimentelle Onkologie und Therapieforschung, Technische Universität München, Munich, Germany; ${ }^{3}$ Institut für Allgemeine Pathologie und Pathologische Anatomie, Technische Universität München, Munich, Germany; ${ }^{4}$ Abteilung für Gefässchirurgie, Technische Universität München, Munich, Germany; ${ }^{5}$ Institut für Pharmakologie und Toxikologie, Technische Universität München, Munich, Germany; and ${ }^{6}$ III. Medizinische Klinik und Poliklinik Klinikum rechts der Isar, Technische Universität München, Munich, Germany

There is a need for in vivo monitoring of cell engraftment and survival after cardiac cell transplantation therapy. This study assessed the feasibility and usefulness of combined PET and MRI for monitoring cell engraftment and survival after cell transplantation. Methods: Human endothelial progenitor cells (HEPCs), derived from CD34+ mononuclear cells of umbilical cord blood, were retrovirally transduced with the sodium iodide symporter (NIS) gene for reporter gene imaging by ${ }^{124}$ I-PET and labeled with iron oxides for visualization by MRI. Imaging and histologic analysis were performed on 3 groups of nude rats on days 1, 3, and 7 after intramyocardial injection of 4 million HEPCs. Results: In vitro studies demonstrated stable expression of functional NIS protein and normal viability of HEPCs after transduction. On day 1, after intramyocardial transplantation, iron- and NIS-labeled HEPCs were visualized successfully on $\mathrm{MRI}$ as a regional signal void in the healthy myocardium and on PET as ${ }^{124}$ I accumulation. The ${ }^{124} \mid$ uptake decreased on day 3 and was undetectable on day 7 , and the MRI signal remained unchanged throughout the follow-up period. Histologic analysis with CD31 and CD68 antibodies confirmed the presence of either labeled or nonlabeled control transplanted HEPCs at the site of injection on day 1 but not on day 7 , when only iron-loaded macrophages were seen. Furthermore, deoxyuride-5'-triphosphate biotin nick end labeling showed extensive apoptosis at the site of transplantation. Conclusion: The combination of MRI and PET allows imaging of localization and survival of transplanted HEPCs together with morphologic information about the heart. Although iron labeling rapidly loses specificity for cell viability because of phagocytosis of iron particles released from dead cells, reporter gene expression provided specific information on the

Received Dec. 1, 2008; revision accepted Mar. 16, 2009.

For correspondence or reprints contact: Takahiro Higuchi, Nuklearmedizinische Klinik und Poliklinik der Technischen Universität München, Klinikum rechts der Isar Ismaninger, Strasse 22, 81675 Munich, Germany.

E-mail: higuchi@po2.nsknet.or.jp

${ }^{*}$ Contributed equally to this work.

COPYRIGHT @ 2009 by the Society of Nuclear Medicine, Inc. number of surviving cells. This multimodality approach allows complementary analysis of cell localization and viability.

Key Words: cardiology; PET; MRI; NIS; cell transplantation; gene expression imaging

J Nucl Med 2009; 50:1088-1094

DOI: 10.2967/jnumed.108.060665

\section{A} lthough myocardial cell transplantation appears to be a promising therapy for cardiac regeneration after myocardial infarction, recent results from large clinical trials have failed to demonstrate major favorable effects in patients $(1,2)$. Efficiency of engraftment and subsequent survival of cells in target tissue are likely to have a major impact on the success of cell transplantation therapies. Currently, only a minority of the cells remains in the heart a few days after delivery, and thus, there is a need for an improved understanding of the basic mechanisms of cell-based regenerative therapies $(1,3)$. Noninvasive cell survival monitoring $(4,5)$ by serial MRI and PET may provide novel insights into cell engraftment, proliferation, and survival and help evaluate novel regenerative cell therapies noninvasively.

Recently, several techniques for direct visualization and monitoring of the survival of magnetically or genetically labeled transplanted cells in the heart have been proposed by means of MRI (6-11) and PET (12). MRI is an attractive modality because it allows localization of the transplanted cells at high spatial resolution combined with good anatomic and functional characterization of the injured myocardium without exposure to ionizing radiation $(6,10)$. Compared with MRI of iron-labeled cells, reporter gene imaging of genetically labeled graft cells with PET may provide more specific information on the number of surviving cells (12-14). How- 
ever, no studies to date have applied a direct comparison of both MRI and PET to address the feasibility of multimodality imaging in monitoring cardiac cell therapy. Thus, we combined magnetic cell labeling for MRI and reporter gene labeling for PET to study the feasibility of serial imaging of localization and survival of transplanted human endothelial progenitor cells (HEPCs) in the normal rat myocardium. The imaging signals were correlated with histologic tissue analysis to validate the MRI and PET data.

\section{MATERIALS AND METHODS}

\section{Donor Cell Preparation and Labeling}

HEPCs were isolated, cultured, and expanded as described previously (15-17). Briefly, cord blood mononuclear cells were obtained from healthy newborn donors. CD34+ cells were isolated using magnetic-activated cell sorting (Direct CD34 Progenitor Cell Isolation Kit; Miltenyi Biotec). Cells were cultured and expanded on medium described elsewhere (16) to express endothelial cell markers, including CD31 ( $>90 \%$ positive). The cord blood cells were obtained after the acquisition of informed parental consent, according to guidelines approved by the ethical committee of the Technische Universität München.

VSV-G pseudotyped retroviral vectors expressing reporter genes LacZ encoding $\beta$-galactosidase or human sodium iodide symporter (NIS) were produced by transient transfections (18) after cloning the respective complementary DNAs into pBullet (19). NIS complementary DNA was derived from full-length NIS/pcDNA3 (20). HEPCs were infected with NIS-expressing retroviral vectors and subsequently with LacZ-expressing retroviral vectors the following day. Transduction was performed at passages 3-5.

For in vivo transplantation experiments, the cells were further expanded and labeled with iron oxides directly before injection into animals. Donor cells were labeled with iron oxides by being incubated with $15 \mu \mathrm{L} / \mathrm{mL}$ of medium of Lipofectin (Invitron Limited) and $100 \mu \mathrm{g} / \mathrm{mL}$ of medium of Resovist (28 $\mathrm{mg}$ of iron oxide per milliliter) (Schering) for $2 \mathrm{~h}$ at $37^{\circ} \mathrm{C}$. Subsequently, the cells were washed with a phosphate-buffered saline solution.

\section{Assessment of NIS Reporter Gene Expression and Phenotype of HEPCs}

To detect cell surface expression of NIS, $1 \times 10^{6}$ trypsinized HEPCs were incubated with a monoclonal antibody directed against an extracellular domain of NIS (VJ2; kindly provided by Sabine Costagliola, Free University of Brussels) (21) and an antimouse IgG fluorescein isothiocyanate labeled antibody (Serotec) followed by flow cytometry using a Vantage fluorescenceactivated cell sorter (FACS) (Becton Dickinson) and the CellQuest software (Becton Dickinson). Likewise, CD31 surface expression was confirmed after viral transduction using a fluorescein isothiocyanate labeled monoclonal antibody (Serotec) and the respective IgG1 isotype control as detailed by the manufacturer.

Uptake of radioactive iodide by NIS-labeled HEPCs was studied essentially as described by Spitzweg et al. (22), except that $3,700 \mathrm{~Bq}$ of ${ }^{99 \mathrm{~m} T c}$ were used to label $1 \times 10^{5}$ HEPCs in reaction tubes.

Electrophysiologic recordings of NIS-labeled HEPCs were made using the whole-cell configuration of the patch-clamp technique through previously described methods (23). Currents were recorded with an EPC-9 patch-clamp amplifier (HEKA Electronics) and Pulse (version 8.54) software (HEKA Electronics).
After retroviral NIS transduction, HEPCs were subjected to tube formation assay. A $300-\mu \mathrm{L}$ volume of BD Matrigel basement membrane matrix high-concentration (BD Biosciences) dilution was suspended into 24-well tissue culture plates. After gel formation, $6 \times 10^{4}$ HEPCs or human umbilical venous endothelial cells per well were seeded, and tube formation was observed microscopically $15 \mathrm{~h}$ after seeding.

\section{Animal Studies}

Male athymic nude rats (CRL:NIH-rnu; Charles River Laboratories) weighing 200-230 g were anesthetized with an intramuscular administration of midazolam $(0.1 \mathrm{mg} / \mathrm{kg})$, fentanyl $(1 \mu \mathrm{g} /$ $\mathrm{kg})$, and medetomidine $(10 \mu \mathrm{g} / \mathrm{kg})$ for thoracotomy. The heart was then exposed, and $200 \mu \mathrm{L}$ of HEPCs $\left(4 \times 10^{6}\right.$ cells $)$ were injected into the anterolateral wall of the left ventricle using a 27 -gauge insulin syringe. At this point, the chest was closed, and the animals were allowed to recover until the MRI and PET study.

MRI and PET were performed on day 1 after transplantation of HEPCs labeled with iron $(n=4)$, the NIS reporter gene $(n=4)$, or both iron and the NIS reporter gene $(n=10)$. In a subset of animals $(n=6)$, MRI and PET were repeated on days 3 and 7 after transplantation of HEPCs labeled with both iron and the NIS to monitor cell survival. MRI and PET were done consecutively on the same day using separate anesthesia for each imaging session. The animals were sacrificed after PET for autoradiography and histologic analysis of the heart. To exclude potential effects of labeling and imaging on survival of HEPCs after transplantation, additional control histologic experiments were performed using nonlabeled HEPCs without imaging on days $1(n=3)$ and $7(n=$ 3) after transplantation.

\section{PET and Analysis}

Rats anesthetized with midazolam, fentanyl, and medetomidine were imaged prone on a dedicated small-animal PET scanner. Data acquisition commenced $60 \mathrm{~min}$ after the injection of ${ }^{124} \mathrm{I}$ iodide solution (25-30 MBq obtained from Nuklearmedizinische Klinik, University Essen) into the cannulated tail vein and continued for 30 min to detect NIS-expressing cells. Immediately after this acquisition, ${ }^{13} \mathrm{~N}-\mathrm{NH}_{3}$ was injected intravenously $(50-60 \mathrm{MBq})$. It was allowed to distribute for $5 \mathrm{~min}$, after which a 10-min PET acquisition took place to visualize perfused myocardium. A subset of rats $(n=$ 4) was imaged for $90 \mathrm{~min}$ starting at the time of injection of ${ }^{124} \mathrm{I}$ for dynamic analysis of tracer accumulation. All data were acquired in list mode and were graphed into sinograms. Sinograms were reconstructed using filtered backprojection with a cutoff at the Nyquist frequency. For quantification of tracer uptake, a region of interest was placed manually at the site of cell injection in a transverse slice of the mid ventricle, and uptake values were expressed as mean percentage injected dose per cubic centimeter.

\section{MRI and Analysis}

The rats were anesthetized as previously described and were imaged prone on a clinical MRI system (Achieva, 1.5-T; Philips) equipped with a dedicated small-animal electrocardiographic triggering system (SA Instruments Inc.) and a single-loop microscopy coil (Philips).

After initial scout scanning, 2-dimensional cine short-axis views of the left ventricle were obtained using an electrocardiographically triggered segmented gradient-echo technique with the following imaging parameters: matrix, $512 \times 512$; repetition time, $11.5 \mathrm{~ms}$; echo time, $4.4 \mathrm{~ms}$; in-plane resolution, $0.3 \times 0.3 \mathrm{~mm}$; slice thickness, $2 \mathrm{~mm}$; and temporal resolution, $11.5 \mathrm{~ms}$. 
For quantification of the iron signal (signal void), regions of interest were defined manually at the cell injection site and at a contralateral site in the normal wall of a mid-ventricular slice. Contrast-to-noise ratio was calculated using $\left(\mathrm{SI}_{\mathrm{myo}}-\mathrm{SI}_{\text {cell }}\right) / 0.5 \times$ $\left(\mathrm{SD}_{\text {myo }}+\mathrm{SD}_{\text {cell }}\right)$, where $\mathrm{SI}$ is signal intensity, myo is normal myocardium, and cell is cell injection site.

\section{Autoradiography, Histology, and Immunohistochemistry}

The rats were sacrificed immediately after PET, and the hearts were excised, frozen, and embedded in methylcellulose. Serial short-axis cryosections 20 and $5 \mu \mathrm{m}$ thick were obtained for autoradiography and histology, covering the entire heart at 1-mm intervals using a cryostat (HM500OM microtome; Micrim). Tracer distribution was determined by analysis of the digitized autoradiographs (PhosphorImager 445 SI; Molecular Dynamics). Regions of interest were manually defined in a region of focal tracer uptake and in a contralateral normal region in a midmyocardial section. If no focal myocardial tracer accumulation was observed, a region of interest was placed in the anterolateral wall. Radioactivity values of each region of interest were recorded as background-corrected photostimulated luminescence per square millimeter and were expressed as uptake ratio, which was calculated by dividing the value of the focal tracer uptake region by that of the contralateral normal area.

Histologic and immunohistochemical staining was performed using standard techniques. Ex vivo detection of LacZ gene expression of the donor cells was done with 5-bromo-4-chloro3-indolyl-b-D-galactoside (X-gal) and eosin staining. The presence and localization of iron particles were assessed by Prussian blue and eosin staining. Monoclonal mouse antihuman platelet endothelial cell adhesion molecule-1 antibodies (CD31, clone JC70A, 1:40 dilution; DAKO) were used to detect HEPCs in the rat heart, and monoclonal mouse antirat CD68 antibodies (1:100 dilution; Abcam Limited) were used to detect macrophages. For the detection of apoptotic cells, deoxyuride-5'-triphosphate biotin nick end labeling (TUNEL) (Fluorescent Cell Death Detection
Kit; Roche) was used according to the instructions of the manufacturer. Staining were done on serial sections, except for dual staining of iron and macrophages in the same tissue sections by Prussian blue and CD68 immunohistochemistry (macrophages) based on alkaline phosphatase staining, resulting in a red color.

\section{Statistical Analysis}

All results were expressed as mean \pm SD. Statistical analysis was done with StatMate III (ATMS Co., Ltd.). Continuous variables were compared by the unpaired Student $t$ test, and multiple groups were compared by ANOVA using ranks (KruskalWallis test) followed by the Dunn multiple-contrast hypothesis test to identify differences in each group. A value of $P$ less than 0.05 was considered statistically significant.

\section{RESULTS}

\section{In Vitro Analysis of Cell Labeling}

Serial FACS analyses indicated that HEPCs stably expressed surface NIS protein, because $50.5 \% \pm 6.9 \%$ of $51.0 \% \pm 5.3 \%$ cells remained positive after 6 passages. The NIS was functional as evidenced by ${ }^{99 \mathrm{~m}} \mathrm{Tc}$ uptake by the HEPCs (Figs. 1A and 1B). The electrophysiologic properties of HEPCs are shown in Figures 1C and 1D. Wild-type HEPCs (control a), uninfected fraction of HEPCs (control b), and NIS-labeled HEPCs did not differ in their baseline electrophysiology, measured as current-voltage relationships in the absence of $\mathrm{I}^{-}$(Fig. 1C). Figure 1D shows that the addition of 10 or $50 \mathrm{mM}^{-} \mathrm{I}^{-}$to the bathing solution caused a concentration-dependent inward current of 40 and $280 \mathrm{pA}$, respectively, in only HEPCs expressing NIS. The effect was reversible and reproducible.

Analysis of growth kinetics revealed no differences in the early phase and only slight retardation of growth and onset of cellular senescence, which reduced the total
FIGURE 1. (A) Increased $99 \mathrm{mTC}$ uptake was demonstrated after retroviral NIS gene transduction. (B) ${ }^{99 m}$ Tc-uptake correlated well with percentage of NISpositive cells as determined by FACS analysis. (C) Results of electrophysiologic characterization. Current was recorded from control HEPCs (control a), uninfected HEPCs derived from transduced pool (control b), or hNIS-expressing HEPC (NIS). Graph shows no difference in current-voltage relationships between control and NIS cells at baseline. (D) Cells were perfused with $\mathrm{I}^{-}$in bathing solution at indicated concentrations. Representative currents from uninfected cells (control HEPC) and NIS-positive cells (NIS labeled) are shown. Current was recorded at $V_{h}=-50 \mathrm{mV}$. Superfusion of $\mathrm{I}^{-}$induced inward current only in NIS-expressing HEPC, indicating functionality of NIS protein.
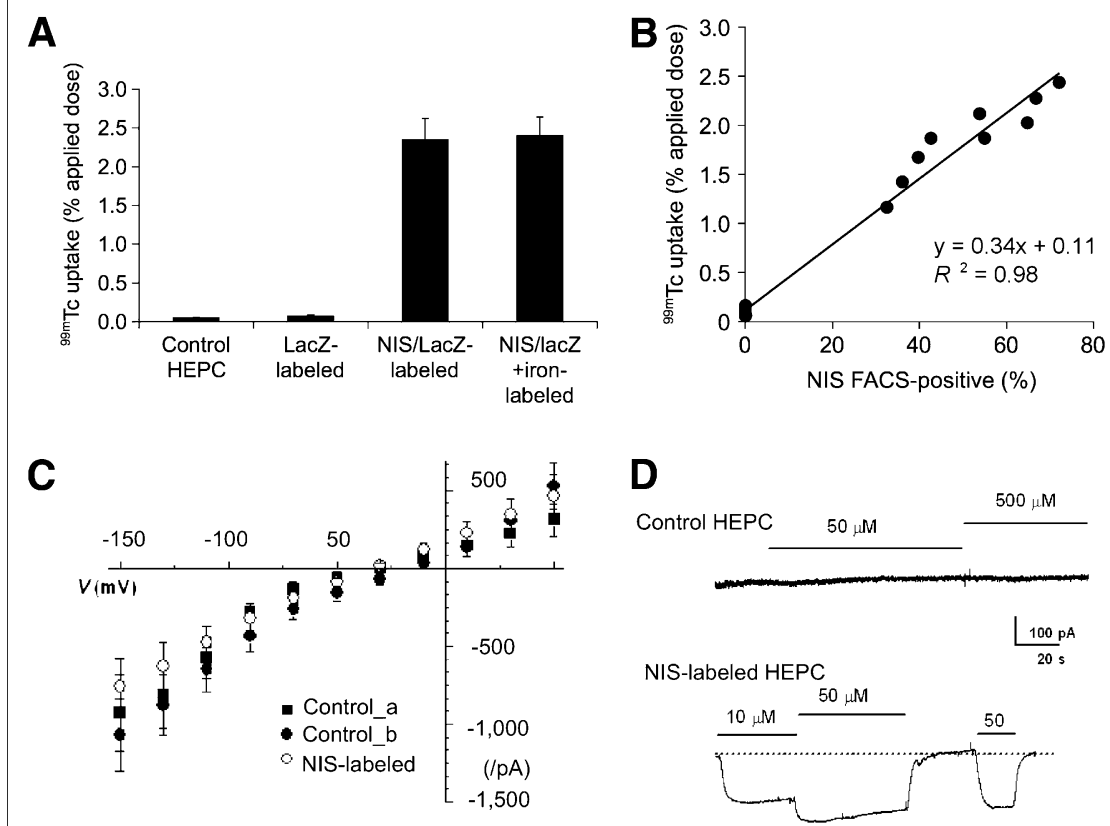

D

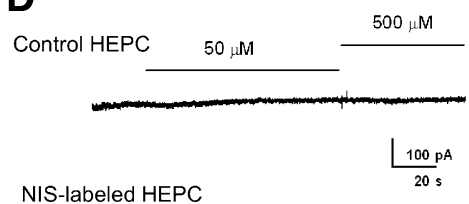

NIS-labeled HEPC

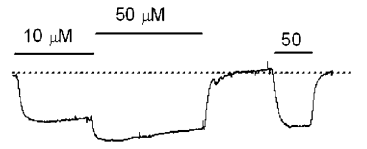


number of cumulative cells by a factor of 62 after NIS transduction, compared with nontransduced cells. The infection procedure did not change the endothelial phenotype of HEPCs, since both parental HEPCs $(94.2 \%)$ and retrovirally transduced HEPCs (94.8\%) stained positively for HEPC surface marker CD31 in FACS analysis. Likewise, NIS-labeled HEPCs were positive in the tube formation assay, indicating their potential to contribute to vessel formation (Supplemental Fig. 1A; supplemental materials are available online only at http://jnm.snmjournals.org).

The effectiveness of iron labeling was shown by Prussian blue staining to be more than 90\% (Supplemental Fig. 1B). FACS analysis of iron oxide-labeled cells revealed that labeling does not change the surface expression of NIS (NIS expression was $50.1 \%$ vs. $50.5 \%$ on day 3 and $58.3 \%$ vs. $61.1 \%$ on day 7 in the absence vs. presence, respectively, of iron labeling). Likewise, the functionality of the NIS protein on the HEPC was not altered after iron labeling. ${ }^{99 \mathrm{~m}} \mathrm{Tc}$ uptake was $2.4 \% \pm 0.3 \%$ of the applied dose in the absence of iron labeling and $2.4 \% \pm 0.2 \%$ in the presence of iron labeling on day 3 after labeling, and similar values were obtained $7 \mathrm{~d}$ after labeling $(2.1 \% \pm$ $0.2 \%$ vs. $2.8 \% \pm 0.1 \%$ in the absence vs. presence, respectively, of iron labeling). Additionally, there was no difference in the cumulative number of viable cells between iron-labeled cells and nonlabeled control cells.

\section{In Vivo Cell Monitoring by MRI and PET}

Representative MR and PET images of transplanted HEPCs labeled with iron only, NIS only, or both iron and NIS are shown in Figure 2A. The injection site was readily visualized as a signal void in T2*-weighted MR images on day 1 after transplantation in all rats that received HEPCs labeled with iron. Similarly, all rats that received HEPCs labeled with the NIS reporter gene demonstrated a clearly visualized focal ${ }^{124} \mathrm{I}$ accumulation in the chest on day 1. Fusion of ${ }^{124} \mathrm{I}$ and ${ }^{13} \mathrm{~N}-\mathrm{NH}_{3}$ perfusion images confirmed the localization of focal ${ }^{124}$ I uptake at the site of intramyocardial injection in the anterolateral wall. The localization of the MRI signal void and the PET-detected ${ }^{124} \mathrm{I}$ accumulation agreed well on day 1 after transplantation in all rats that received HEPCs labeled with both iron and NIS reporter. Autoradiography of myocardial tissue sections confirmed strong, focal uptake of ${ }^{124} \mathrm{I}$ on day 1 after transplantation of HEPCs labeled with NIS, LacZ, and iron particles. Uptake of ${ }^{124} \mathrm{I}$ corresponded to the site of iron particles and LacZ gene expression in serial sections studied by autoradiography, Prussian-blue staining, and $\mathrm{X}$-galactosidase staining (Fig. 2B).

Dynamic PET revealed that ${ }^{124} \mathrm{I}$ accumulation peaked at 10 min after tracer injection and then washed out slowly (Fig. 2C). At the time of imaging, 60-90 min after injection, approximately $65 \%$ of peak activity was still present in the myocardium. Blood-pool activity decreased rapidly, providing the best contrast for imaging myocardial uptake 45 min after tracer injection.

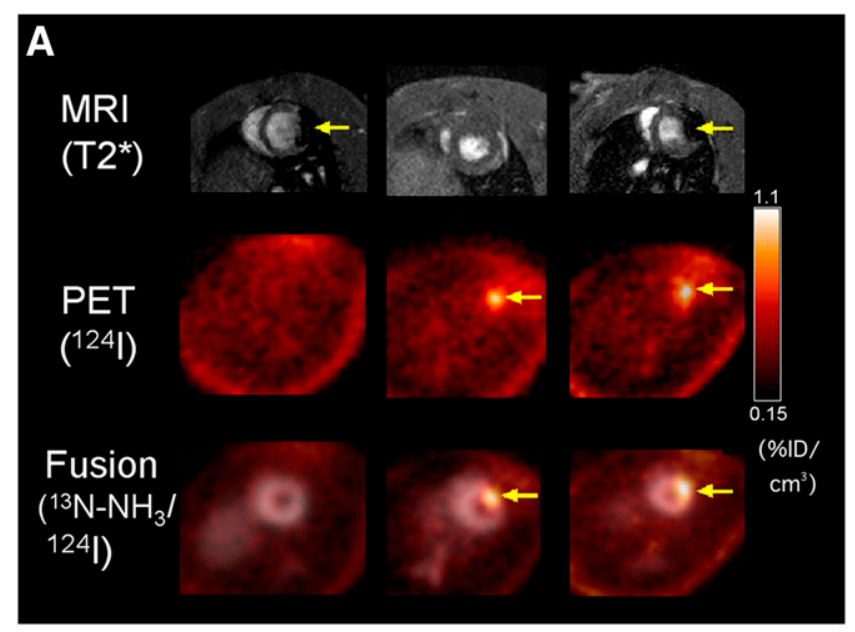

B
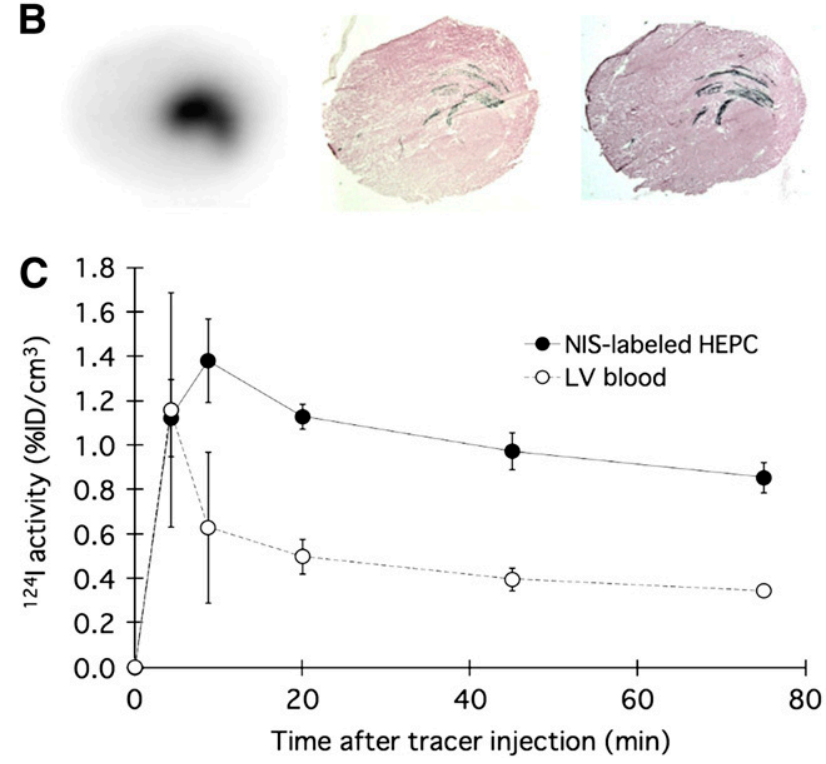

FIGURE 2. (A) Representative MR and PET images of rat thorax $1 \mathrm{~d}$ after cell transplantation. Donor cells were labeled with iron (left), NIS only (middle), or both iron and NIS (right). T2*-weighted MR images are shown in top row, ${ }^{124}$ I-PET images in middle row, and fused images with ${ }^{13} \mathrm{~N}-\mathrm{NH}_{3}$ (gray scale) and ${ }^{124}$ (color scale) in bottom row. Rat with ironlabeled HEPCs showed signal void at myocardium by MRI, whereas rat with HEPCs expressing NIS demonstrated focal ${ }^{124}$ I accumulation by PET. (B) Examples of consecutive myocardial sections of rat heart on day 1 after transplantation of HEPCs labeled with iron oxides, NIS reporter, and LacZ reporter. Autoradiography for ${ }^{124}$ I uptake mediated by NIS reporter (left), X-galactosidase staining for LacZ gene expression of graft cells (middle), and Prussian blue staining for iron particle detection (right) are seen in corresponding locations. (C) Mean ( \pm SD) time-activity curves after ${ }^{124} \mid$ administration of transplanted cell and left ventricular blood measured by PET $(n=4)$.

The results of serial MRI and PET on days 1, 3, and 7 after transplantation of HEPCs labeled with both iron and NIS reporter are shown in Figures 3A and 3B. The MRI signal void observed on day 1 persisted until day 7 . In contrast, ${ }^{124}$ I uptake detected on day 1 rapidly decreased and was not detectable on 
FIGURE 3. (A) Results from longitudinal imaging of transplanted HEPCs labeled with both iron oxides and NIS gene. Representative short-axis images of rat on days 1,3 , and 7 after transplantation. (B) Time course of mean $( \pm \mathrm{SD}) \mathrm{MRI}$ and PET signal. Contrastto-noise ratio by $\mathrm{MRI}$ at site of cell injection was stable over $7 \mathrm{~d}$, but ${ }^{124}$ I uptake decreased rapidly and was not detectable on day 7 after cell engraftment $\left({ }^{\star} P<0.001\right)$. (C and D) Results of autoradiography confirmed changes in ${ }^{124}$ I signal by PET. Representative autoradiograph $(\mathrm{C})$ and bar graph of mean $( \pm S D){ }^{124}$ I uptake ratio (D) demonstrate rapid decrease of uptake signal as early as $3 \mathrm{~d}$ after transplantation $\left({ }^{\star} P<0.001\right)$.

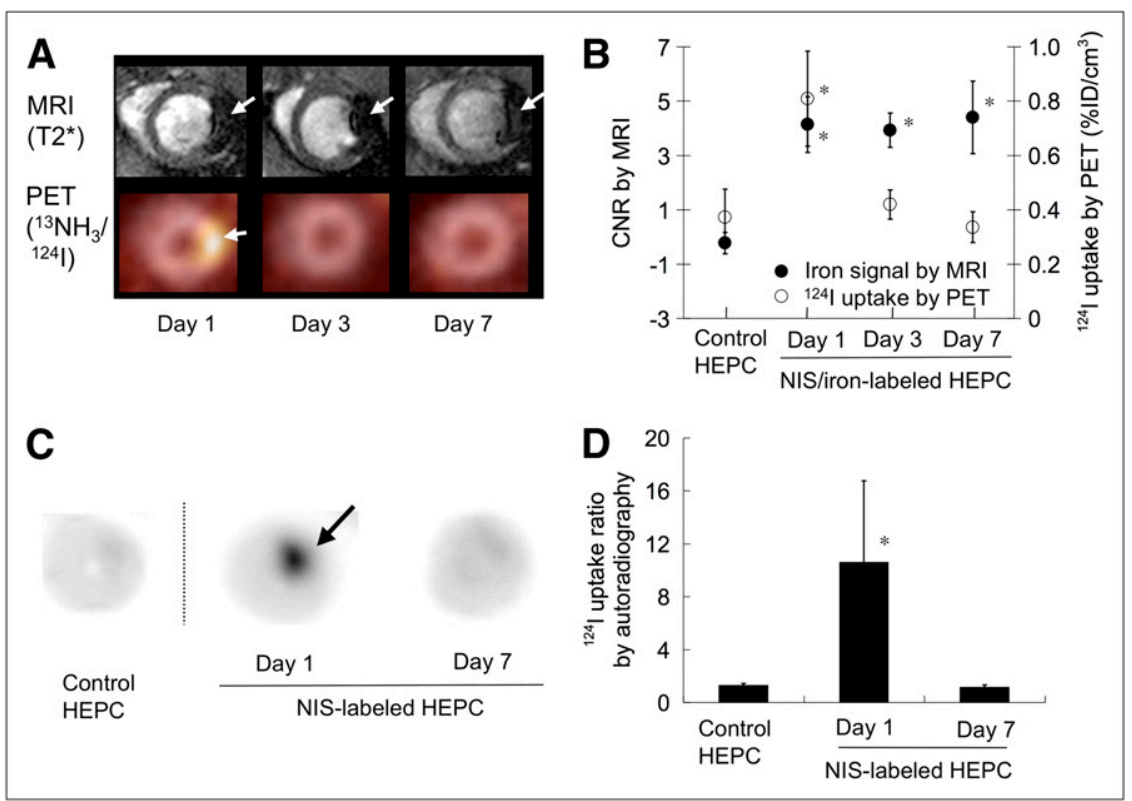

day 7. Autoradiography confirmed a rapid decrease of ${ }^{124} \mathrm{I}$ accumulation on days 3 and 7 (Figs. 3C and 3D).

\section{Histopathologic Examination}

Results were obtained using either labeled or nonlabeled HEPCs. Staining with human CD31 antibodies revealed large numbers of HEPCs at the site of injection on day 1 and their absence on day 7 (Supplemental Fig. 2). On day 1, TUNEL staining demonstrated considerable numbers of apoptotic nuclei colocalizing with the transplanted HEPCs in serial sections. These findings are consistent with the rapid death of HEPCs after transplantation in normal rat myocardium. According to the study with transplantation of nonlabeled control HEPCs, labeling the cells does not result in cell death.

Histologic detection of iron particles is shown in Figure 4. Prussian blue staining revealed that iron particles colocalized with CD31-positive HEPCs on day 1 after transplantation of HEPCs labeled with iron. However, iron deposition was still detected at the injection site despite the absence of HEPCs on day 7 after transplantation. Only a few CD68-positive macrophages were seen on day 1 , but their number increased by day 7. On day 7 , the remaining iron particles colocalized with macrophages as seen by dual staining with Prussian blue and CD68 immunohistochemistry of the same tissue sections (Supplemental Fig. 3). These results indicate retention of the iron particles in macrophages after graft cell death.

\section{DISCUSSION}

To our knowledge, this study was the first to demonstrate the feasibility of combined MRI and PET to investigate the location and survival of transplanted HEPCs in the myocardium after dual labeling with reporter gene and iron oxide particles.

The NIS protein is an intrinsic transmembrane glycoprotein, which mediates active transport of iodide and sodium across the cytoplasmic membrane (24). Because it is not naturally expressed in the heart, it is a suitable reporter gene for cardiac applications. There are some potential advantages of NIS over other reporter genes, such as viral thymidine kinase (25). First, the NIS protein is expressed in the thyroid gland naturally and, thus, is not immunogenic and does not produce toxic byproducts. Second, apart from the ${ }^{124} \mathrm{I}$ PET probe, ${ }^{123} \mathrm{I},{ }^{131} \mathrm{I}$, and ${ }^{99 \mathrm{~m} T c}$ are also available as alternative imaging probes for SPECT and do not require complex probe synthesis $(25,26)$. In addition, compared with thymidine kinase reporter gene imaging, the target-to-background ratios were higher (27), rendering this approach more sensitive for in vivo cell detection. In this study, HEPCs were stably transduced to express functional NIS on the cell surface. Our analyses revealed no evidence of toxicity after NIS transduction. Transduction did not alter expression of surface markers such as CD31, growth kinetics, or the ability of cells to form tubes of Matrigel. Furthermore, expression of NIS did not alter the basic electrophysiologic properties of the cells. Endothelial cells are nonexcitable, and thus voltage-dependent ion channels are sparse (28). Accordingly, the addition of increasing concentrations of iodide induced an inward current only in the NIS-expressing cells, indicating functionality of the symporter in HEPCs. Additional labeling of the HEPCs with iron oxides affected neither expression of NIS nor cell phenotype and growth. These cell-labeling techniques may be well suited for future clinical approaches to cell therapy, although further careful work is necessary to establish the long-term effects on cell viability, function, and differentiation.

MRI visualized the localization of iron-labeled HEPCs exactly in the left ventricular myocardium $1 \mathrm{~d}$ after intramyocardial injection. Although iron signal and location 

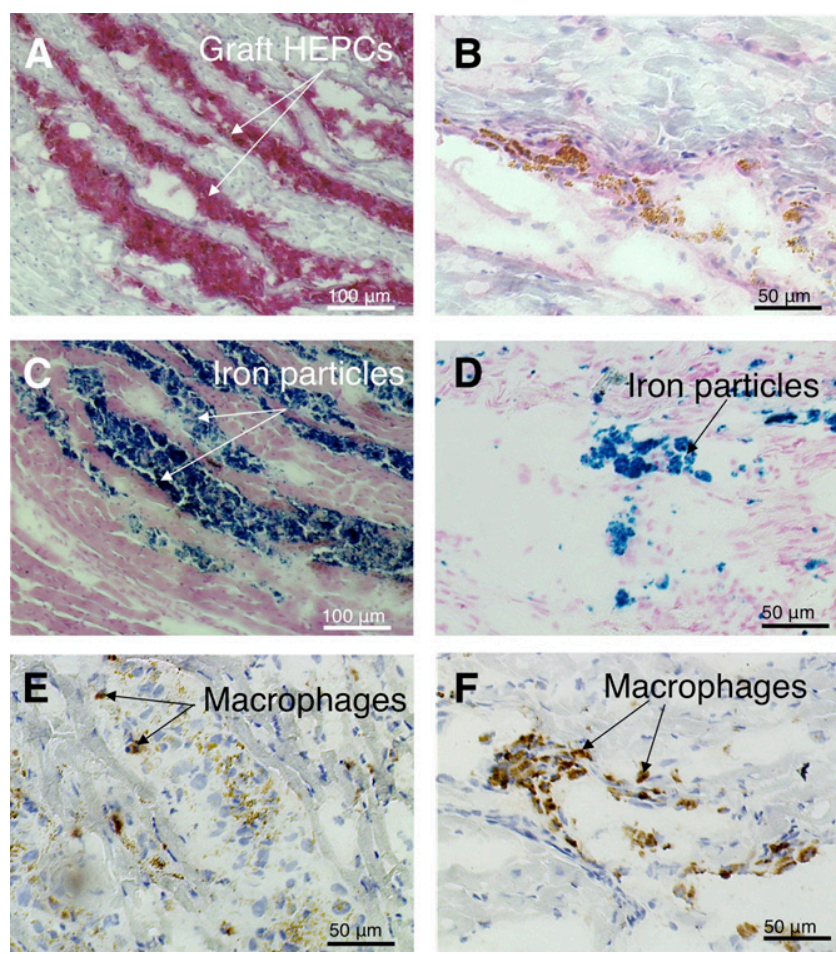

FIGURE 4. Histologic detection of iron particles after transplantation of iron-labeled HEPCs in rat hearts. Representative images of human CD31 immunohistochemical analysis for detection of graft HEPCs on days 1 (A) and 7 (B), Prussian-blue iron staining on days 1 (C) and 7 (D), and CD68 immunohistochemical macrophage analysis on days 1 (E) and 7 (F) are shown. Localization of iron particles and graft HEPCs was observed on day 1 at site of cell transplantation ( $A$ and $C$ ). However, iron particles remained in heart on day 7 , despite absence of graft HEPCs (B and D). On day 7 , increased number of macrophages $(F)$ was found.

remained unchanged until $7 \mathrm{~d}$ after engraftment by MRI, ${ }^{124}$ I uptake signal by PET decreased rapidly and was not detectable on day 7. In agreement with these observations, postmortem analysis showed rapid death of the graft cells and retention of the iron particles in macrophages on day 7 . These results indicate that only reporter gene PET was specific for the presence of viable HEPCs. Accordingly, the dual imaging approach would provide initial localization of transplanted cells and ventricular morphology by MRI, whereas reporter gene PET signal would add specific information on the number of viable transplanted cells.

MRI is the most accurate method to evaluate left ventricular function and can also provide myocardial infarct size - an important surrogate endpoint for the evaluation of cardiac cell therapies (29). Reporter gene PET signals have been compared with fused MR images to localize stem cells after injection (30). Recently, direct visualization of iron oxide-labeled cells using MRI was also demonstrated $(10,31)$. Because this concept is valid early after transplantation, and labeling of cells is simple, it has been used for tracking graft cells in several studies $(6,32)$. However, a limitation of magnetic cell-labeling methods has been demonstrated by this study using HEPCs, serial MRI, PET, and histologic examinations. Although the iron signal observed by MRI on day 1 did not differ from that on day 7, rapid loss of HEPCs after transplantation was demonstrated by CD31 and TUNEL staining. Histologic analysis indicated that the iron particles were still present at the site of injection but had localized in macrophages. This finding is consistent with results recently reported by Amsalem et al. demonstrating that the MRI signal from iron-labeled rat mesenchymal stem cells transplanted into infarcted myocardium remained present throughout the 4-wk follow-up whereas histology demonstrated that transplanted cells remained present for only $1 \mathrm{wk}$ after transplantation (8). As in our study, iron-positive macrophages were seen at 4 wk (8). Although MRI of iron-labeled cells may be helpful in precisely localizing and studying the efficiency of delivery of transplanted cells in the early phase, these results point to the need to determine the exact time course of cell death after transplantation in each application by complementary methods.

Conceptually, the reporter gene-encoding products will be continuously expressed as long as the cell is viable, even after cell division, provided that stable transduction of the gene has been achieved $(4,12,14,33)$. In our study, the ${ }^{124} \mathrm{I}$ signal of NIS reporter PET cell imaging decreased below the detection limit of PET rapidly, $3 \mathrm{~d}$ after cell transplantation. The most likely explanation is a rapid loss of viable graft HEPCs as indicated by human CD31 immunohistochemical analysis and TUNEL staining. However, ${ }^{124} \mathrm{I}$ uptake also depends on stable expression of the NIS protein on viable cells and on efficient retention of the probe. In our study, these were confirmed by FACS analyses and dynamic PET, respectively. Although adenoviral gene expression is efficient in many cell types, this vector type does not integrate and, thus, does not allow for sustained gene transfer in dividing cells. To obtain stable gene expression, we chose retroviral vectors as an integrating system. In this system, reporter gene expression is driven by the long terminal repeat promoter, and epigenetic silencing of expression may become a problem in the long term. Deregulated premalignant cell proliferation (34) represents another possible limitation of retroviral vectors in a clinical study. To this end, new, potentially safer vectors have become available, including self-inactivating vectors that allow for use of insulators and nonviral promoters (35). Further development of safe and efficient vectors and promoters is an important task for the clinical use of reporter gene cell imaging.

Intramyocardial transplantation of HEPCs derived from $\mathrm{CD} 34+$ mononuclear cells has been reported to increase capillary density and ventricular function in a rat model of myocardial infarction $(16,36)$. Our study indicates that only small numbers of cells remain in the heart more than $1 \mathrm{~d}$ after transplantation. In a test tube, in the absence of any background detection, the limit of our assay was $1 \times 10^{4}$ cells, and in vivo, $1 \times 10^{6}$ cells could be detected at the site of injection after $2 \mathrm{~h}$. Thus, lower numbers of HEPCs may 
still have been present despite the absence of PET signal. Although our study demonstrated that HEPCs were successfully visualized by both MRI and PET in the healthy myocardium, future studies should evaluate these methods for visualization of stem cells transplanted in the infarcted or periinfarcted myocardium, which is the most important target of cell therapy and may modify cell survival.

\section{CONCLUSION}

MRI of iron-labeled cells provides a readily available tool for noninvasive cell localization early (1 d) after transplantation, but cell death and subsequent phagocytosis of free iron by macrophages render the MRI signal nonspecific for tracking the implanted HEPCs later. In contrast, reporter gene imaging was demonstrated to have good specificity for tracking graft cell survival and thus may be more attractive for evaluation of therapies aimed at maximizing graft cell survival. The combination of both techniques may provide a useful tool for the simultaneous monitoring of cell survival by PET and early cell engraftment, as well as improvement in left ventricular function by MRI.

\section{ACKNOWLEDGMENTS}

We thank Renate Hegenloh for performing immunohistology and Sabine Costagliola, Free University of Brussels, for providing anti-hNIS antibody. We are grateful to Betina Wagner, Sybille Reder, Axel Weber, Marc Huisman, and Axel Martínez-Möller for their technical assistance. We also thank the technologists of the cyclotron and MRI units of the TU München for helping with the experiments, and we are grateful to Gola Javadi for her careful editorial assistance. This project was funded by the Deutsche Forschungsgemeinschaft (Be2217/4-1 and -2), by the EU EC-FP6-project DiMI (LSHB-CT-2005-512146), and by the EU Clinigene Network of Excellence (LSHB-CT-2006-018933).

\section{REFERENCES}

1. Welt FG, Losordo DW. Cell therapy for acute myocardial infarction: curb your enthusiasm? Circulation. 2006;113:1272-1274.

2. Bartunek J, Vanderheyden M, Wijns W, et al. Bone-marrow-derived cells for cardiac stem cell therapy: safe or still under scrutiny? Nat Clin Pract Cardiovasc Med. 2007;4(suppl 1):S100-S105.

3. Oettgen P, Boyle AJ, Schulman SP, Hare JM. Cardiac stem cell therapy: need for optimization of efficacy and safety monitoring. Circulation. 2006;114:353-358.

4. Beeres SL, Bengel FM, Bartunek J, et al. Role of imaging in cardiac stem cell therapy. J Am Coll Cardiol. 2007;49:1137-1148.

5. Frangioni JV, Hajjar RJ. In vivo tracking of stem cells for clinical trials in cardiovascular disease. Circulation. 2004;110:3378-3383.

6. Hill JM, Dick AJ, Raman VK, et al. Serial cardiac magnetic resonance imaging of injected mesenchymal stem cells. Circulation. 2003;108:1009-1014.

7. Himes N, Min JY, Lee R, et al. In vivo MRI of embryonic stem cells in a mouse model of myocardial infarction. Magn Reson Med. 2004;52:1214-1219.

8. Amsalem Y, Mardor Y, Feinberg MS, et al. Iron-oxide labeling and outcome of transplanted mesenchymal stem cells in the infarcted myocardium. Circulation. 2007;116(11, suppl)I38-I45.

9. Bulte JW, Kostura L, Mackay A, et al. Feridex-labeled mesenchymal stem cells: cellular differentiation and MR assessment in a canine myocardial infarction model. Acad Radiol. 2005;12(suppl 1):S2-S6.

10. Kraitchman DL, Heldman AW, Atalar E, et al. In vivo magnetic resonance imaging of mesenchymal stem cells in myocardial infarction. Circulation. 2003; 107:2290-2293.
11. Kraitchman DL, Tatsumi M, Gilson WD, et al. Dynamic imaging of allogeneic mesenchymal stem cells trafficking to myocardial infarction. Circulation. 2005; 112:1451-1461.

12. Wu JC, Chen IY, Sundaresan G, et al. Molecular imaging of cardiac cell transplantation in living animals using optical bioluminescence and positron emission tomography. Circulation. 2003;108:1302-1305.

13. Krishnan M, Park JM, Cao F, et al. Effects of epigenetic modulation on reporter gene expression: implications for stem cell imaging. FASEB J. 2006; 20:106-108

14. Wu JC, Spin JM, Cao F, et al. Transcriptional profiling of reporter genes used for molecular imaging of embryonic stem cell transplantation. Physiol Genomics. 2006;25:29-38.

15. Jaffe EA, Nachman RL, Becker CG, Minick CR. Culture of human endothelial cells derived from umbilical veins: identification by morphologic and immunologic criteria. J Clin Invest. 1973;52:2745-2756.

16. Ott I, Keller U, Knoedler M, et al. Endothelial-like cells expanded from CD34+ blood cells improve left ventricular function after experimental myocardial infarction. FASEB J. 2005;19:992-994.

17. Herder C, Tonn T, Oostendorp R, et al. Sustained expansion and transgene expression of coagulation factor VIII-transduced cord blood-derived endothelial progenitor cells. Arterioscler Thromb Vasc Biol. 2003;23:2266-2272.

18. Anton M, Wagner B, Haubner R, et al. Use of the norepinephrine transporter as a reporter gene for non-invasive imaging of genetically modified cells. J Gene Med. 2004;6:119-126.

19. Schaft N, Willemsen RA, de Vries J, et al. Peptide fine specificity of antiglycoprotein $100 \mathrm{CTL}$ is preserved following transfer of engineered TCR alpha beta genes into primary human T lymphocytes. J Immunol. 2003;170:21862194.

20. Smanik PA, Liu Q, Furminger TL, et al. Cloning of the human sodium iodide symporter. Biochem Biophys Res Commun. 1996;226:339-345.

21. Pohlenz J, Duprez L, Weiss RE, Vassart G, Refetoff S, Costagliola S. Failure of membrane targeting causes the functional defect of two mutant sodium iodide symporters. J Clin Endocrinol Metab. 2000;85:2366-2369.

22. Spitzweg C, Scholz IV, Bergert ER, et al. Retinoic acid-induced stimulation of sodium iodide symporter expression and cytotoxicity of radioiodine in prostate cancer cells. Endocrinology. 2003;144:3423-3432.

23. Hamill OP, Marty A, Neher E, Sakmann B, Sigworth FJ. Improved patch-clamp techniques for high-resolution current recording from cells and cell-free membrane patches. Pflugers Arch. 1981;391:85-100.

24. Dai G, Levy O, Carrasco N. Cloning and characterization of the thyroid iodide transporter. Nature. 1996;379:458-460.

25. Chung JK, Kang JH. Translational research using the sodium/iodide symporter in imaging and therapy. Eur J Nucl Med Mol Imaging. 2004;31:799-802.

26. Shin JH, Chung JK, Kang JH, et al. Feasibility of sodium/iodide symporter gene as a new imaging reporter gene: comparison with HSV1-tk. Eur J Nucl Med Mol Imaging. 2004;31:425-432.

27. Miyagawa M, Anton M, Wagner B, et al. Non-invasive imaging of cardiac transgene expression with PET: comparison of the human sodium/iodide symporter gene and HSV1-tk as the reporter gene. Eur J Nucl Med Mol Imaging. 2005; 32:1108-1114

28. Nilius B, Droogmans G. Ion channels and their functional role in vascular endothelium. Physiol Rev. 2001;81:1415-1459.

29. Higuchi T, Nekolla SG, Jankaukas A, et al. Characterization of normal and infarcted rat myocardium using a combination of small-animal PET and clinical MRI. J Nucl Med. 2007;48:288-294.

30. Gyöngyösi M, Blanco J, Marian T, et al. Serial noninvasive in vivo positron emission tomographic tracking of percutaneously intramyocardially injected autologous porcine mesenchymal stem cells modified for transgene reporter gene expression. Circ Cardiovasc Imaging. 2008;1:94-103.

31. Bulte JW, Kraitchman DL. Iron oxide MR contrast agents for molecular and cellular imaging. NMR Biomed. 2004;17:484-499.

32. He G, Zhang $\mathrm{H}$, Wei $\mathrm{H}$, et al. In vivo imaging of bone marrow mesenchymal stem cells transplanted into myocardium using magnetic resonance imaging: a novel method to trace the transplanted cells. Int J Cardiol. 2007;114:4-10.

33. Bengel FM, Schachinger V, Dimmeler S. Cell-based therapies and imaging in cardiology. Eur J Nucl Med Mol Imaging. 2005;32(suppl 2):S404-S416.

34. Hacein-Bey-Abina S, Von Kalle C, Schmidt M, et al. LMO2-associated clonal T cell proliferation in two patients after gene therapy for SCID-X1. Science. 2003;302:415-419.

35. Nienhuis AW, Dunbar CE, Sorrentino BP. Genotoxicity of retroviral integration in hematopoietic cells. Mol Ther. 2006;13:1031-1049.

36. Kawamoto A, Tkebuchava T, Yamaguchi J, et al. Intramyocardial transplantation of autologous endothelial progenitor cells for therapeutic neovascularization of myocardial ischemia. Circulation. 2003;107:461-468. 